This item was submitted to Loughborough's Research Repository by the author.

Items in Figshare are protected by copyright, with all rights reserved, unless otherwise indicated.

\title{
HIP-KNEE control for gait assistance with Powered Knee Orthosis
}

PLEASE CITE THE PUBLISHED VERSION

http://dx.doi.org/10.1109/ROBIO.2013.6739554

\section{PUBLISHER}

(c) IEEE

VERSION

AM (Accepted Manuscript)

\section{PUBLISHER STATEMENT}

This work is made available according to the conditions of the Creative Commons Attribution-NonCommercialNoDerivatives 4.0 International (CC BY-NC-ND 4.0) licence. Full details of this licence are available at: https://creativecommons.org/licenses/by-nc-nd/4.0/

\section{LICENCE}

CC BY-NC-ND 4.0

\section{REPOSITORY RECORD}

Lai, Wai-Yin, Hao Ma, Wei-Hsin Liao, Daniel Tik-Pui Fong, and Kai-Ming Chan. 2019. "HIP-KNEE Control for Gait Assistance with Powered Knee Orthosis”. figshare. https://hdl.handle.net/2134/21200. 


\title{
HIP-KNEE Control for Gait Assistance with Powered Knee Orthosis
}

\author{
Wai-Yin Lai, Hao Ma, Wei-Hsin Liao, Daniel Tik-Pui Fong, and Kai-Ming Chan
}

\begin{abstract}
A Powered Knee Orthosis (PKO) was recently developed for the elderly and patients with disordered gait to regain normal walking. In order to enhance the PKO performance and reduce system complexity especially for people with muscle weakness in their knee joints, an algorithm named HIP-KNEE control is proposed. This algorithm is based on the analysis of kinematic gait model, and the desired knee joint angle (KNEE) is estimated from the measurements of hip joint angle (HIP). The relationship between HIP and KNEE is modeled as a polynomial, which can be easily implemented to an embedded controller for real-time control. This control method is natural to subjects with good function in hip joint, and it can provide help in walking without special training. An Inertia Measurement Units (IMU) is used for obtaining HIP input, and integrated with a footswitch for checking the heel condition, the gait assistance performance can be further improved.
\end{abstract}

\section{INTRODUCTION}

Because of aging problem and some diseases, more people with muscle weakness in their lower extremities demand solutions to assist their daily motions [1]. Different powered Lower Extremity Exoskeletons (LEEs) were designed to deal with this great issue. They can provide assistive force/torque at the wearers' joints, mainly for actuating wearer's hip and knee joint motions in the sagittal plane, like HAL [2], ReWalk [3], Esko [4], and Vanderbilt Exoskeleton [5]. HAL is hybrid controlled with surface electromyography (SEMG) and grounded reaction force (GRF) for the motion intention recognition; ReWalk, Esko, and Vanderbilt Exoskeleton are LEEs designed for lower extremity paralysis and controlled by observing the wearer's weight shift.

In contrast to those group of severe patients requesting full suit LEEs, people with dysfunction in a single or some joints are suggested to use powered orthosis designed for specific joint(s), so that would bring out merits in terms of weight, size, performance, and cost. In our group, a Powered Knee Orthosis (PKO) was especially designed for the elderly and patient with dysfunction in knee joint [6]. Limited gait information and actuator configuration make traditional LEE control, like utilizing inverse dynamics, more difficult to be implemented in a 1-DOF device. Hence, the sensing and control design for PKO should be able to take advantage of partial gait kinematics measured at the leg wearing PKO.

In this research, we propose a PKO control algorithm for gait assistance based on the relationship between HIP and KNEE in the sagittal plane during normal walking. The

W. Y. Lai, H. Ma, and W. H. Liao are with the Department of Mechanical and Automation Engineering, The Chinese University of Hong Kong, Hong Kong SAR, China.\{wylai2, hma, whliao\}@mae.cuhk.edu.hk. D. T. P. Fong and K. M. Chan are with the Department of Orthopaedics \& Traumatology, The Chinese University of Hong Kong, Hong Kong SAR, China.dfong@ort.cuhk.edu.hk,kaimingchan@cuhk.edu.hk. objective of gait assistance is to provide a good reference gait pattern in knee joint to let the wearer follow. By measuring the HIP during walking, the target KNEE for normal walking is estimated and used as the control input of the actuator.

\section{HIP-KNEE RELATIONSHIP IN NORMAL GAIT}

\section{A. Gait Kinematics}

Human gait on the level ground is a periodic motion with specific hip and knee joint angle patterns in each gait cycle. Based on the standard clinical gait analysis (CGA) results [7], the normalized HIP and KNEE angles in the sagittal plane with respect to gait cycle percentage are shown in Fig. 1. Here, HIP is the hip joint angle between thigh and gravity vector, and KNEE is the knee joint angle between shank and thigh. Then we further calculate the derivative of the hip angular velocity with respect to gait cycle percentage and plot it into the same figure. Actually, the normalized hip angular velocity is the product of hip angular velocity in time domain, denoted as HIP', and the gait period $T$.

The normal gait cycle can be divided into two phases: swing phase (about $0 \sim 60 \%$ ) and stance phase (about $60 \sim 100 \%$ ) [8]. Based on Fig. 1, we can find that the KNEE and HIP' curves have similar pattern in the swing phase. When KNEE is plotted as a function of HIP' in Fig. 2, a linear estimation, shown as the dashed line, can be used to represent the HIP' and KNEE relationship in the swing phase approximately.

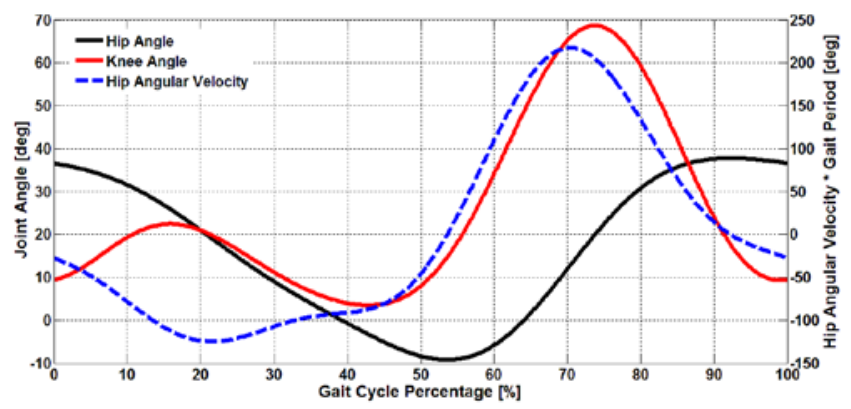

Fig. 1. KNEE and HIP' curves in a normal gait cycle

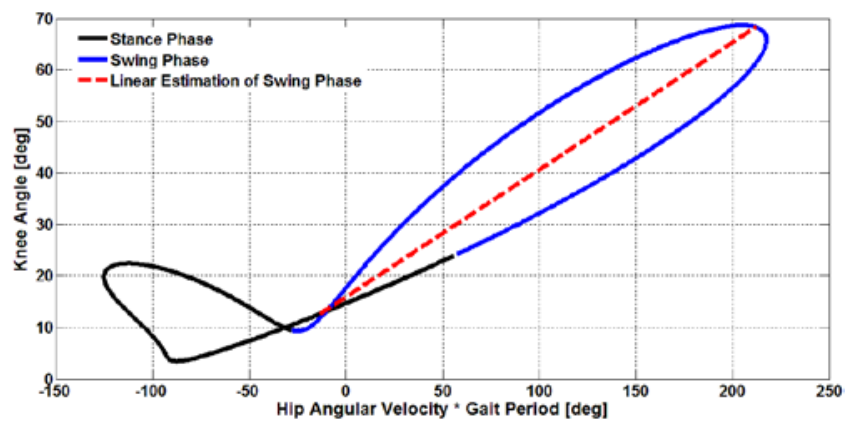

Fig. 2. KNEE and HIP' relationship with linear estimation in swing 


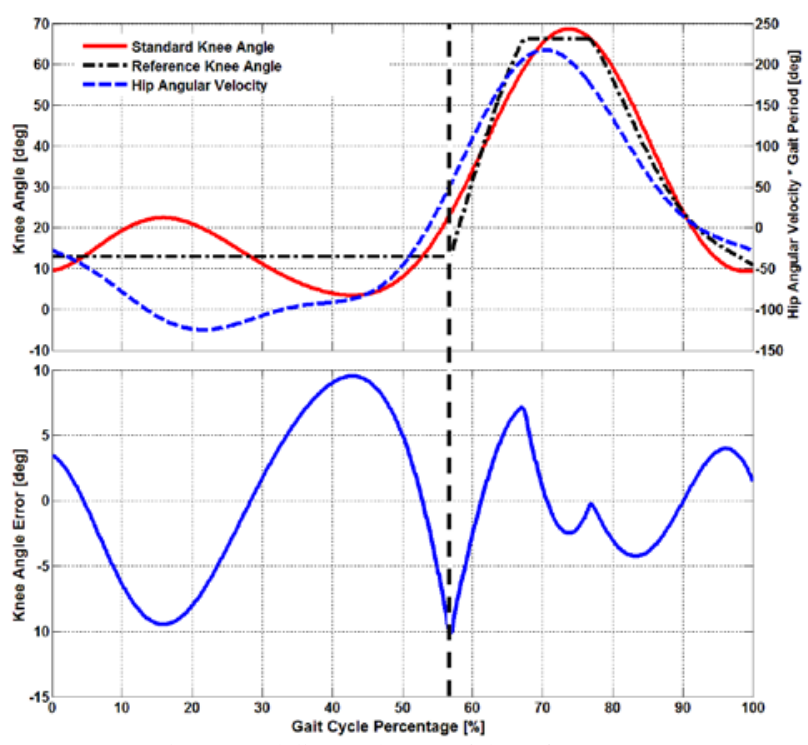

Fig. 3. Modeling and error of the reference KNEE

\section{B. Model Parameters}

For more accurate swing phase KNEE modeling, a linear function of both HIP and HIP' with saturation given in (1) is further considered.

$$
\theta_{\text {knee }}= \begin{cases}\theta_{\text {max }} & \theta_{\text {knee }} \geq \theta_{\text {max }} \\ a * \dot{\theta}_{\text {hip }}+b * \theta_{\text {hip }}+c & \text { Otherwise } \\ \theta_{\text {min }} & \theta_{\text {knee }} \leq \theta_{\text {min }}\end{cases}
$$

Least square method is used for minimizing the error between model and standard CGA data in the swing phase. The optimized model parameters are: $a=28.86 * T, b=$ $0.44, c=3.57^{\circ}, \theta_{\max }=66.24^{\circ}, \theta_{\min }=12.74^{\circ}$, where $T$ is the gait period. The correlation coefficient in the swing phase is higher than 0.98. The result reference KNEE is shown in Fig. 3 with corresponding error. The maximum error in the swing phase is about $10^{\circ}$, and the average error is $3.3^{\circ}$. Here, we just consider the stance phase in the reference KNEE as constant, and this will be explained later in the HIP-KNEE control section.

\section{Model Verification}

After obtained the optimized parameters of the gait model, the HIP-KNEE model is further verified by analyzing another set of CGA data from a young healthy subject. Subject performed level ground walking at the speed of about $1.4 \mathrm{~m} / \mathrm{s}$, and CGA data is collected at $100 \mathrm{~Hz}$ frequency. Based on the collected gait data, reference KNEE is calculated off-line and shown in Fig. 4. It can be seen that the generated KNEE is similar to the actual KNEE, and the correlation coefficient is more than 0.95 in the swing phase.

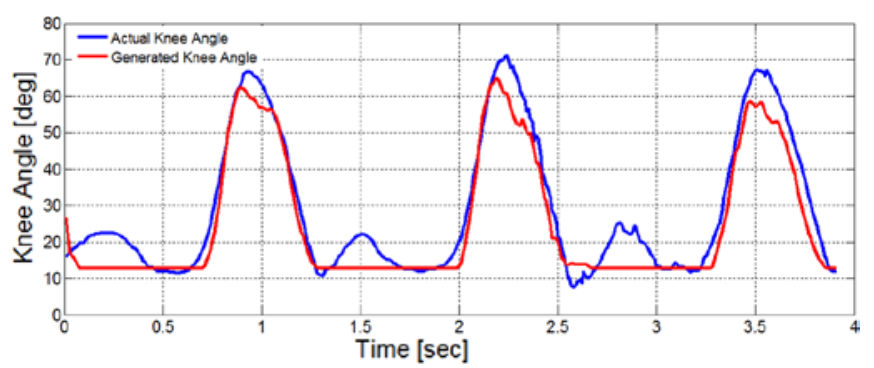

Fig. 4. HIP-KNEE model verification with a young healthy subject
It proves that the proposed HIP-KNEE model could generate good knee reference based on hip joint measurements. We will later use this model in the proposed HIP-KNEE control method.

\section{PKO SYSTEM DESIGN}

Considering the suggestions from physicians and the design of other LEEs, we wish to make our PKO design as compact and light as possible and place it around knee joint with least attachment [9]. Therefore, the design of PKO is constrained with less measurable gait information and controllable aspects than the LEEs, e.g., the ground reaction information, other joint angles, etc. Nevertheless, the sensors at thigh and shank can provide information of HIP, KNEE and the interaction force.

With an IMU sensor placed at the thigh link, calibrated beforehand, 3-axis HIP can be obtained directly with $100 \mathrm{~Hz}$ update rate. It therefore gets rid of using attachment to the hip joint and allows free motion of the wearer's hip joint. The thigh link and the shank one are attached to wearer's thigh and shank with tailor-made knee orthosis. A geared motor with encoder is used to actuate knee joint for controlling the KNEE. In addition, a footswitch is placed at the ankle part to detect the foot-ground contact condition. The overall PKO scheme is presented in Fig. 5.

1) Mechanical design: The PKO mechanical design is presented in Fig. 6. A geared motor (Maxon RE35 $24 \mathrm{~V}$ ) is employed to actuate the knee joint with synchronous belt gear train. The maximum angular velocity is $2.8 \mathrm{rad} / \mathrm{s}$ at $16.8 \mathrm{~V}$ with a maximum torque of $118.4 \mathrm{Nm}$, which is large enough for actuating normal gait (peak torque about $60 \mathrm{Nm}$ ) [8]. Tensioners are added to increase belt tension for avoiding belt slippery under high torque transmission. A pair of mechanical stoppers are used to limit the KNEE working range within $0^{\circ}-120^{\circ}$ to ensure safety. Carbon fiber rods are used to construct a light weight and rigid frame, and they

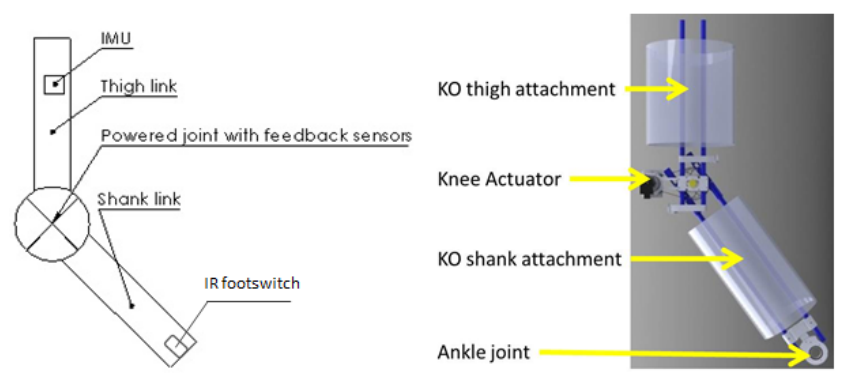

Fig. 5. Scheme of PKO

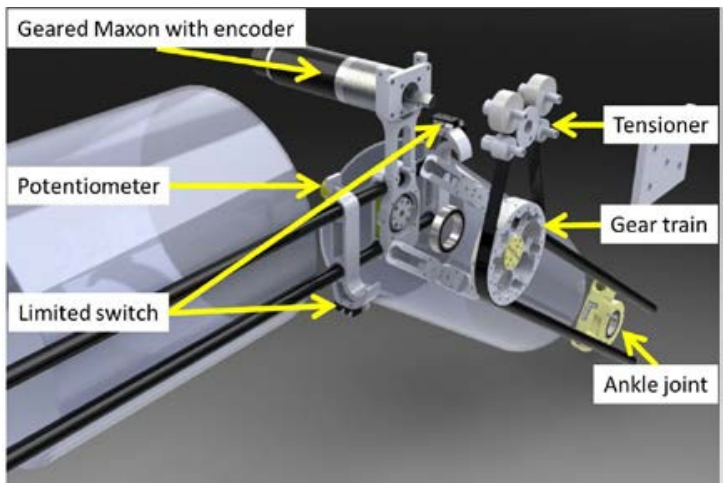

Fig. 6. Mechanical design of PKO 


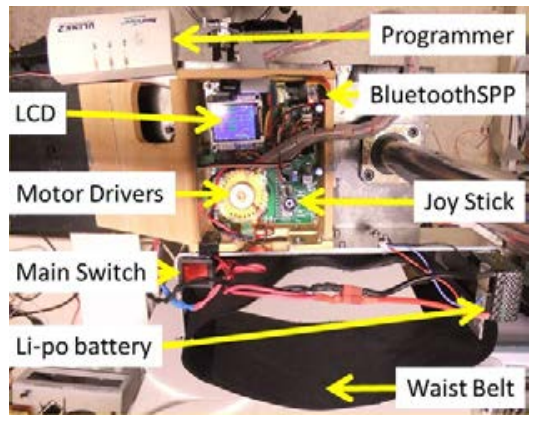

Fig. 7. Embedded controller of PKO on the waist belt

can also be used to adapt ground station and optional ankle joint. The total weight of the mechanical part is about $2.4 \mathrm{~kg}$.

2) Embedded controller: The embedded controller mounted on the waist belt is shown in Fig. 7. The waist belt weighed $840 \mathrm{~g}$ in total is for both sharing the pay load and user interface. The PKO system is powered by 1 to 2 pieces of $7.4 \mathrm{~V}$ lithium polymer batteries whose voltage varies from 7.4 to $16.8 \mathrm{~V}$. For compensating the variance in the voltage source, dynamic $\mathrm{P}$ gain, inversing to the input voltage, is used for maintaining the motor voltage output constant.

3) Sensor system: For the IMU sensor measuring the HIP, a 6-axis MPU6050 combining a 3-axis accelerometer and 3 -axis gyroscope is used. It is set with full range of $\pm 2 \mathrm{~g}$ and $\pm 2000^{\circ} / \mathrm{sec}$. The resolution after conversion and filtering is up to $0.1^{\circ}$. Sensor fusion is adopted to solve the problem of high-frequency noise in accelerometer and low-bias in gyroscope. The footswitch, an infrared proximity sensor, is put at the ankle joint for detecting the contact condition between foot and ground as well as measuring the gait period. The adjustable distance threshold is set to be the condition when heel leaves the ground with angle between foot and ground larger than $15^{\circ}$. KNEE is measured by the integration of a potentiometer and an encoder at the knee joint. The resolution of KNEE is $0.01^{\circ}$. Moreover, a pair of contact switches placed next to mechanical stoppers are used for detecting KNEE motion range as well.

4) Grounded station and optional footpad: As the HIP-KNEE control requires HIP as input, we designed a grounded station with hip actuator shown in Fig. 8. It allows the PKO to be plugged into for preliminary testing. In addition to the grounded station, a footpad is optional to plug-in as the ankle part, which can help support PKO weight from the ground during the stance phase.

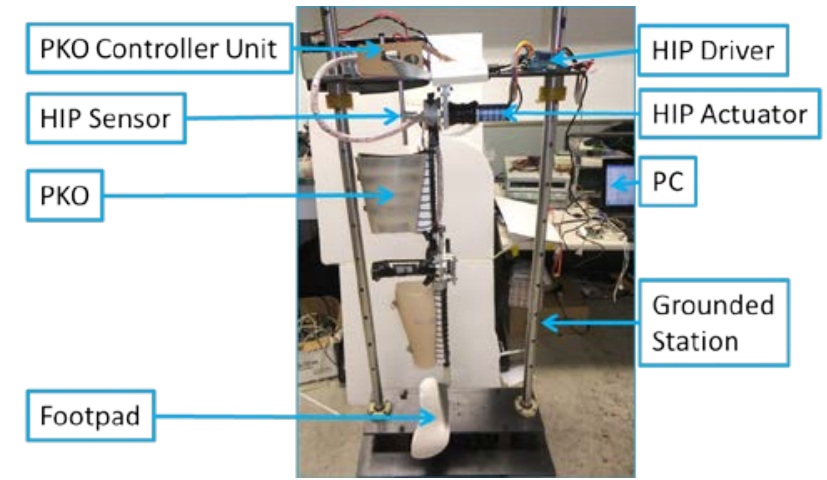

Fig. 8. Grounded station with dummy hip joint for PKO testing

\section{HIP-KNEE CONTROL}

\section{A. Controller Design}

Based on the previous analysis of HIP and KNEE relationship in normal walking, we can design the so called HIP-KNEE control. In the implementation, HIP and HIP' are measured by the IMU sensor, and they are used to generate the reference KNEE for closed-loop PID control during walking. This constraint of this control method is that the wearer should have normal function in the hip joint, so that he/she would provide a good HIP input leading to a normal KNEE output.

In the stance phase, the main function of PKO is to provide a rigid support to the body weight during hip propagation [10]. So in this phase, the KNEE outputs a constant value from the actuator for position locking as shown in the reference KNEE in Section II. However, during the swing phase the PKO should provide a normal flexion and extension swing pattern in KNEE for driving the wearer knee joint.

\section{B. HIP-KNEE Control with Footswitch}

Due to the motor inertia and control performance, the actual KNEE of PKO usually lags the generated reference, which results in a late swing in the swing phase. This problem may affect the gait pattern under control to be a slightly abnormal. In order to deal with this, the function of footswitch is further evaluated.

During walking with $\mathrm{PKO}$, we find that the heel off signal takes place about $10 \%-20 \%$ gait cycle percentage earlier than the KNEE flexion. This is consistent with the CGA data as the heel has to first leave the ground, named terminal stance, before the leg swings. Thus, an early trigger of swing motion can then be realized by the integration of footswitch.

An improved HIP-KNEE control with footswitch is then proposed with advance swing. The control flow chart is given in Fig. 9. During the stance phase, KNEE is locked at constant angle. After the footswitch detects heel off-ground, the heel trigger condition is satisfied and then gait enters swing phase. Swing phase is divided into flexion and extension by reset trigger condition. And reset trigger has three conditions: ankle position in $\mathrm{X}$ direction $\left(\mathrm{X}_{\text {ankle }}\right)$ is larger than zero, HIP is larger than $11^{\circ}$ (the value at heel strike in the standard CGA data), and HIP' is larger than zero. Here, $\mathrm{X}_{\text {ankle }}$ is calculated by using forward kinematic from the hip position. When taking thigh as $386 \mathrm{~mm}$ and shank as 496 mm, it can be calculated as (2).

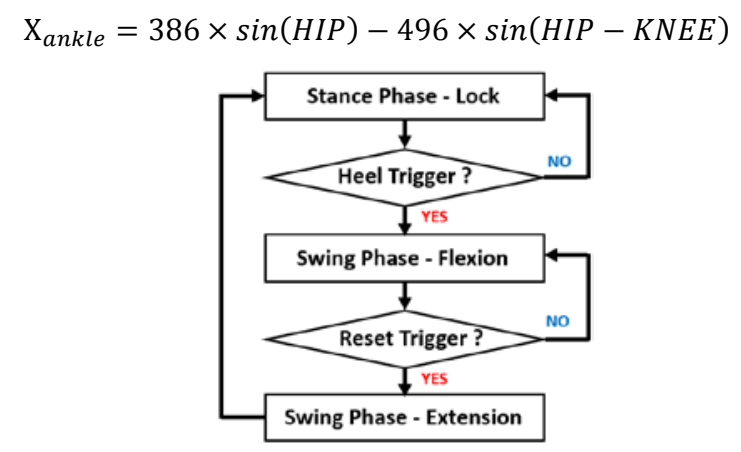

Fig. 9. Flow chart of HIP-KNEE control with footswitch 


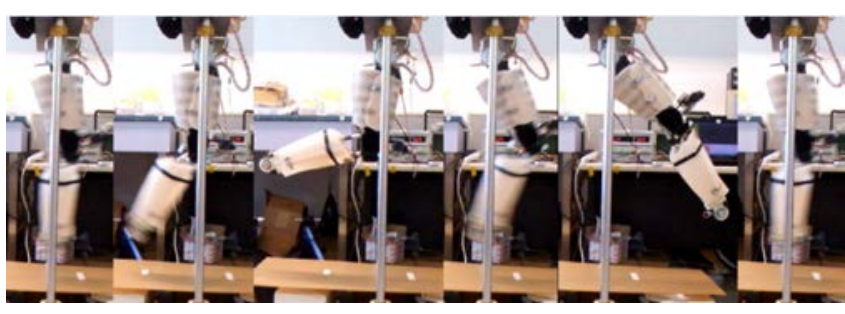

Fig. 10. Emulation of HIP-KNEE control

\section{EXPERIMENT AND EVALUATION}

\section{A. Emulation}

Before subject testing, emulations on the grounded station are conducted to check the performance under feedback PID control as shown in Fig. 10. During emulation, the HIP is controlled by hip actuator with standard HIP from CGA data, and then KNEE is generated online for control according to the HIP-KNEE model. In the emulation, two methods of HIP-KNEE control are performed under different gait period. The results are shown in Figs. 11 and 12 with performance comparison summarized in Table I.

1) HIP-KNEE control without footswitch: Testing with different gait periods are conducted and results are shown in Fig. 11. The generated KNEE reference (KNEE_desire) based on standard HIP is close to the standard KNEE (KNEE_CGA) at all gait periods. However the actual KNEE (KNEE_FB) is tracking the reference with delay. For the fastest walking under $1.6 \mathrm{~s}$ period, the delay at peak is about $0.18 \mathrm{~s}$. This is because the desired speed was faster than the maximum speed of the motor, which is $2.4 \mathrm{rad} / \mathrm{sec}$. In $2.0 \mathrm{~s}$ gait period case, the corresponding performance is even worse due to shaking of HIP actuator at the minima. In $2.4 \mathrm{~s}$ gait period case, the tracking performance is improved to 0.09 s delay as the reference KNEE velocity is close to $2.4 \mathrm{rad} / \mathrm{sec}$ and it is also shaking-free in HIP.

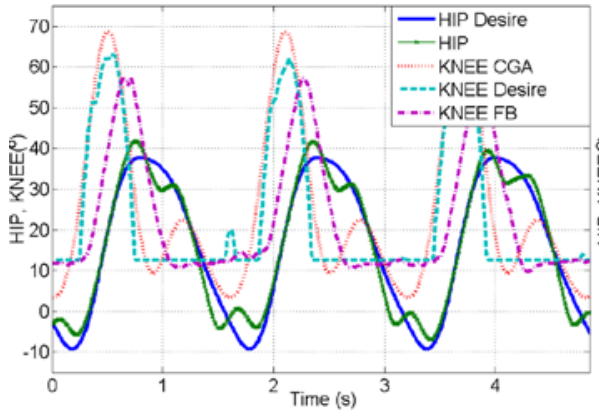

(a)

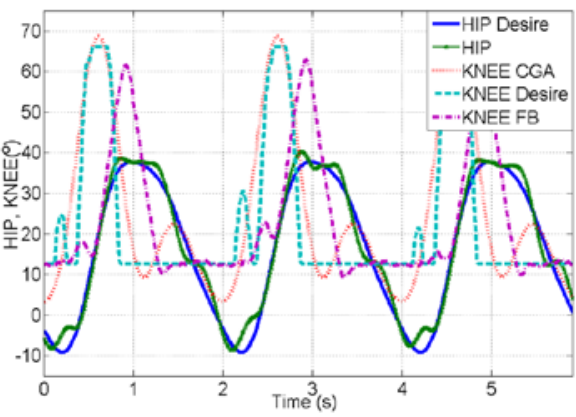

(b)
TABLE I PERFORMANCE COMPARISON IN EMULATION

\begin{tabular}{|c|c|c|c|}
\hline & \multicolumn{3}{|c|}{ Leading standard KNEE \% (KNEE Flexing \%) } \\
\hline Gait Period & $1.6 \mathrm{~s}$ & $2.0 \mathrm{~s}$ & $2.4 \mathrm{~s}$ \\
\hline HIP-KNEE & $-11 \%(86 \%)$ & $-15 \%(91 \%)$ & $-3 \%(98 \%)$ \\
\hline HIP-KNEE+FS & $+12.5 \%(100 \%)$ & $+11.5 \%(100 \%)$ & $+14.6 \%(99 \%)$ \\
\hline
\end{tabular}

This emulation results showed that as long as the reference KNEE velocity is slow enough, the motor can track with good performance. And if there existed a disturbance signal in HIP, the output of HIP-KNEE control would be subject to the fluctuation in swing. For solving the potential disturbing input in HIP and delay output problem, a footswitch can help ease these problems.

2) HIP-KNEE control with footswitch: After sensor fusion with footswitch, results in Fig. 12 showed that the actual KNEE could lead 13\% than the standard KNEE on average, which well-guarantee the actual KNEE can flex to the design flexing angle. And this method is more robust than the original HIP-KNEE control since the unstable tracking in HIP does not directly affect the KNEE output.

From Table I, it can be observed that when the gaiting is slower, the performance would be better in both conditions. When the gait period increases, the HIP-KNEE control with footswitch would maintain a stable and complete flexion with almost constant leading time. Therefore a conclusion can be made that HIP-KNEE control with footswitch can increase the system robust to the gait period and noise in HIP.

\section{B. Subject Testing on Treadmill}

Unlike in the emulation, during subject testing the HIP is generated by the wearer, which may not be exactly the same with standard HIP from CGA data. For better reference, we choose to use a treadmill with preset gait velocity ranging

Fig. 11. Emulation of HIP-KNEE control without footswitch under (a) 1.6s period, (b) 2.0s period, and (c) 2.4s period

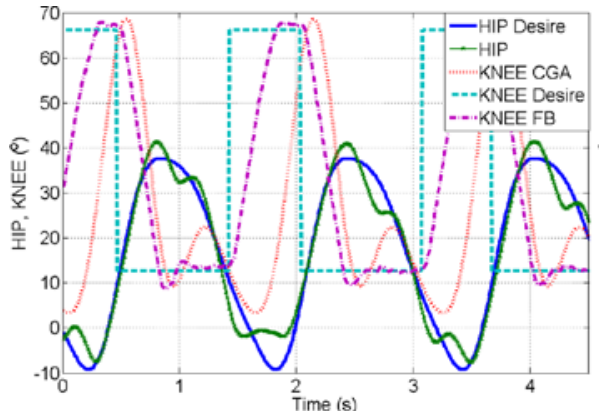

(a)

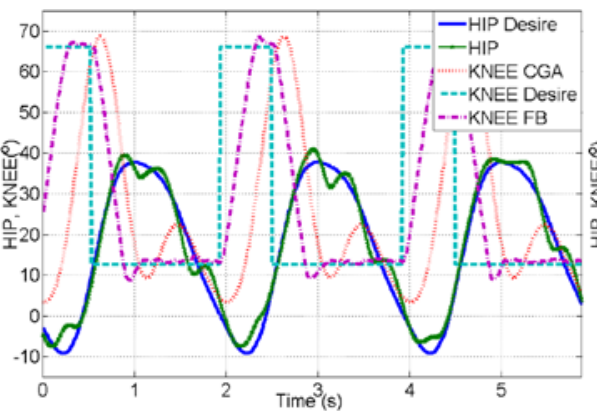

(b)

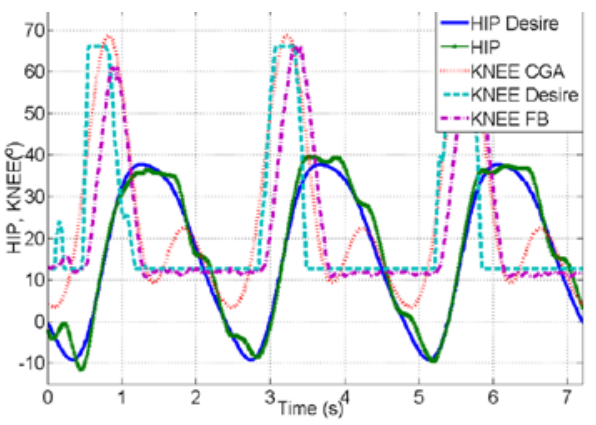

(c)

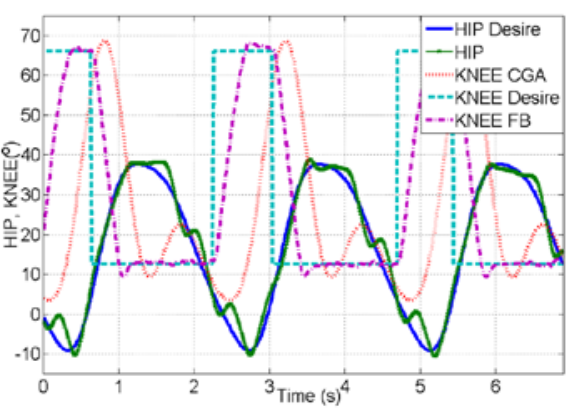

(c)

Fig. 12. Emulation of HIP-KNEE control with footswitch under (a) 1.6s period, (b) 2.0s period, and (c) 2.4s period 


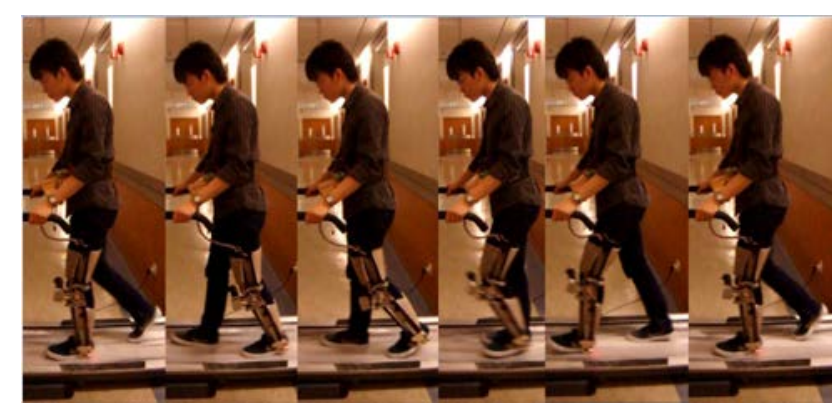

Fig. 13. Subject testing of HIP-KNEE control

from 1 to $2 \mathrm{~m} / \mathrm{s}$. Both HIP-KNEE without footswitch and with footswitch control are tested and compared. A young healthy male subject who can provide a normal motion in the hip joint has enrolled in the subject testing as shown in Fig. 13.

1) HIP-KNEE control without footswitch: From the testing results shown in Fig. 14, the maximum torque provide by $\mathrm{PKO}$ under this control was about $\pm 4.5 \mathrm{Nm}$, while normal gait needs $30-60 \mathrm{Nm}$ at the knee joint. Before both swing, there was brief positive torque, which was drag force. The brief drag duration was increased to the gait velocity. During the swing motion, both flexion and extension were supported by the PKO. The maximum flexing KNEE was about $60^{\circ}$ in $1 \mathrm{~m} / \mathrm{s}$ and $55^{\circ}$ in $2 \mathrm{~m} / \mathrm{s}$.

2) HIP-KNEE control with footswitch: In the testing with footswitch on treadmill, the results are shown in Fig. 15. It can be seen that there was no drag force/torque before the

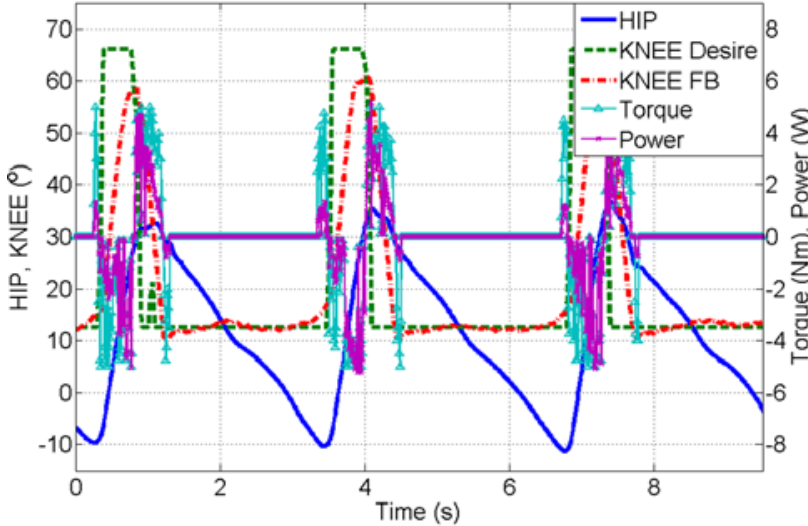

(a)

TABLE II PERFORMANCE COMPARISON IN TORQUE AND TIME

\begin{tabular}{|c|c|c|}
\hline & \multicolumn{2}{|c|}{ Max provided torque and power (Cycle time) } \\
\hline Gait velocity & $1.0 \mathrm{~m} / \mathrm{s}$ & $2.0 \mathrm{~m} / \mathrm{s}$ \\
\hline HIP-KNEE & $4.5 \mathrm{Nm}, 5 \mathrm{~W}(3.21 \mathrm{~s})$ & $4.5 \mathrm{Nm}, 6.5 \mathrm{~W}(1.96 \mathrm{~s})$ \\
\hline HIP-KNEE+FS & $4 \mathrm{Nm}, 6 \mathrm{~W}(2.56 \mathrm{~s})$ & $4 \mathrm{Nm}, 7 \mathrm{~W}(1.63 \mathrm{~s})$ \\
\hline
\end{tabular}

TABLE III PERFORMANCE COMPARISON IN KINEMATIC ASPECT

\begin{tabular}{|c|c|c|}
\hline & \multicolumn{2}{|c|}{ KNEE (HIP) } \\
\hline Gait velocity & $1.0 \mathrm{~m} / \mathrm{s}$ & $2.0 \mathrm{~m} / \mathrm{s}$ \\
\hline HIP-KNEE & $10^{\circ}$ to $60^{\circ}\left(-10^{\circ}\right.$ to $\left.30^{\circ}\right)$ & $10^{\circ}$ to $56^{\circ}\left(-17^{\circ}\right.$ to $\left.36^{\circ}\right)$ \\
\hline HIP-KNEE+FS & $10^{\circ}$ to $60^{\circ}\left(-9^{\circ}\right.$ to $\left.23^{\circ}\right)$ & $10^{\circ}$ to $60^{\circ}\left(-12^{\circ}\right.$ to $\left.21^{\circ}\right)$ \\
\hline
\end{tabular}

swing and purely assistive torque during the swing. The maxima were $\pm 4 \mathrm{Nm}$ and $\pm 4.5 \mathrm{Nm}$ at $1 \mathrm{~m} / \mathrm{s}$ and $2 \mathrm{~m} / \mathrm{s}$ gait velocity. The maximum KNEE flexion was both $60^{\circ}$. The HIP was both moving from $-10^{\circ}$ to $20^{\circ}$.

Comparisons of control performances under two methods are given in Tables II and III. Not limited to the emulation, KNEE flexing under the HIP-KNE control with footswitch is more guaranteed on the treadmill testing among varying velocity. This helps the HIP maintain stable than that without footswitch. As the method with footswitch achieves a faster KNEE velocity, walk would be faster and this would also reduce the required HIP revolution angle for the same speed gait distance at a time.

In Table II, it shows that the HIP-KNEE control and the fusion one with footswitch could generate positive torque to the wearer with different features. First, in the torque aspect,

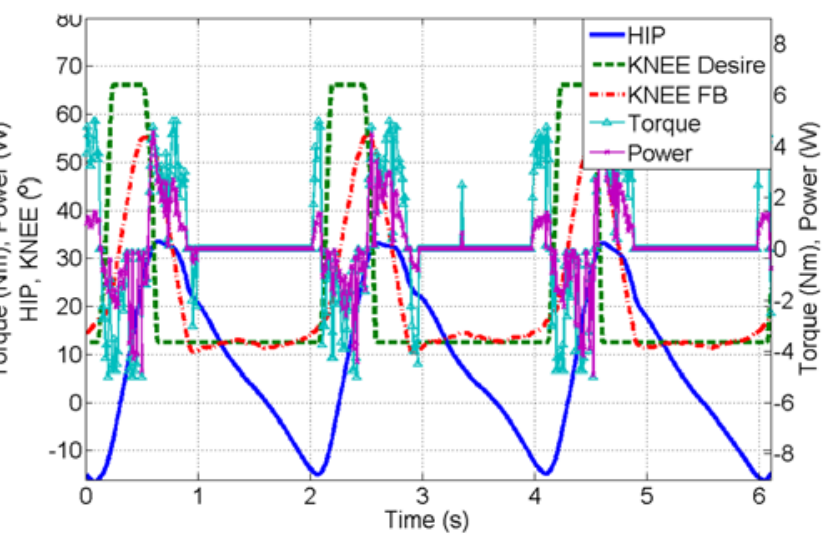

(b)

g. 14. Subject testing of HIP-KNEE control without footswitch under (a) $1 \mathrm{~m} / \mathrm{s}$, (b) $2 \mathrm{~m} / \mathrm{s}$

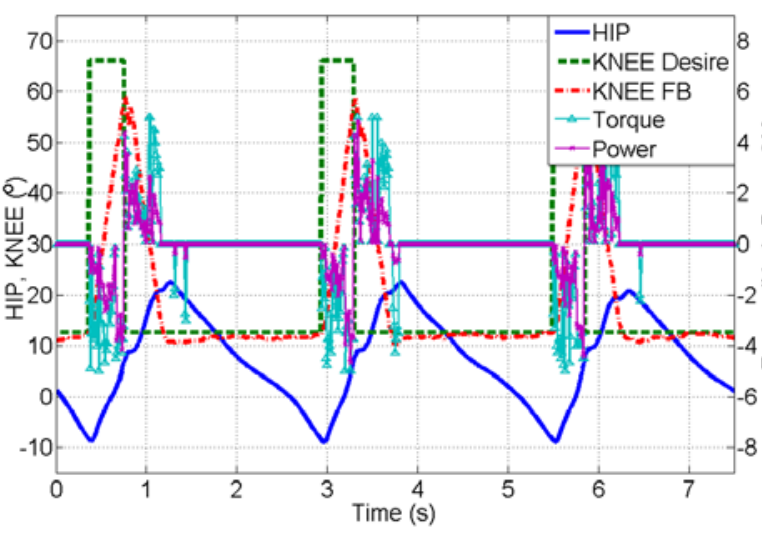

(a)

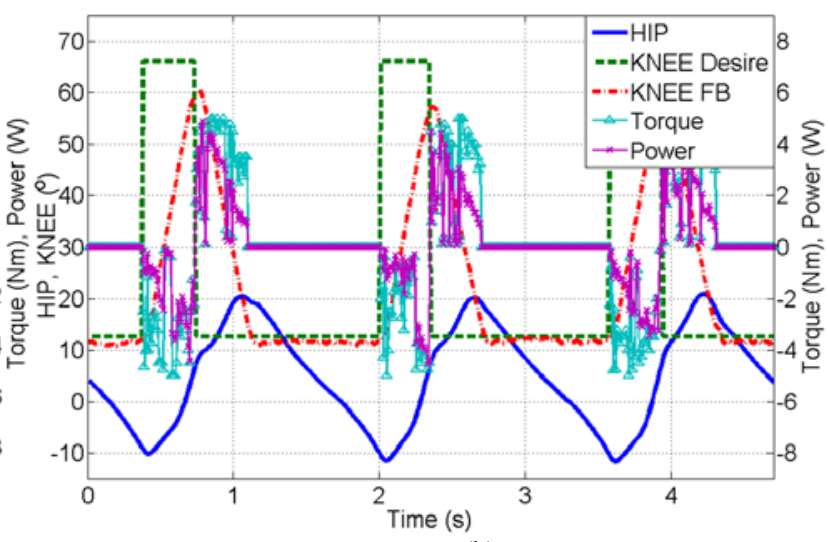

(b)

Fig. 15. Subject testing of HIP-KNEE control with footswitch under (a) $1 \mathrm{~m} / \mathrm{s}$, (b) $2 \mathrm{~m} / \mathrm{s}$ 
the HIP-KNEE method can generate a larger torque than that with footswitch method about $0.5 \mathrm{Nm}$. It may due to the compensation of the brief dragging force. But in the output power, the method with footswitch could deliver a larger power because of a smaller gait period.

A more consistent performance can be found in the HIP-KNEE with footswitch method in the kinematic aspect as shown in Table III. In HIP-KNEE control, the range of motion in both HIP and KNEE varies according to gait velocity, whereas in the method with FS the range of motion of HIP and $\mathrm{KNEE}$ is almost $33^{\circ}$ and $50^{\circ}$ in both velocities.

Overall, results in subject testing showed that both conditions were feasible. However the one with footswitch is more robust to gait velocities and helps the wearer with a steadier gait cycle. The results are consistent with the emulation one to reveal the HIP-KNEE control can improve the performances, ranging from robustness, leading time and providing torque, by using one more FS.

\section{Solutions to Delay Problem}

The KNEE delay is an important issue in the system performance since the user would experience the brief drag due to the delay output. If the user cannot swing the knee, the slower swing motion from the PKO would not pose a significant difference to them. It is because the immobilized patient can regain the mobility by swing HIP to control the disable knee joint. Notwithstanding, if the user can swing the leg, the drag may be sensitive to the one when start to perform knee swing, especially during a fast gait. Drag force was shortly present in the HIP-KNEE control only, and the effect will make the gait cycle slower.

There are solutions to the delay problem in the HIP-KNEE control. Slower motion or HIP-KNEE control with footswitch were discussed previously. First solution is to slow down the motion with a more stabilized HIP input. Because the motor takes time to tracking, slower motion would be easier for it to provide suitable motion. Second solution is using an additional sensor, like footswitch in our case, to receive an earlier signal. Then the IMU becomes one of the auxiliary sensors for the control. This would help a slower and high-torque actuator, which is common in rehabilitation device, to have better control performance.

Another solution that would be more practical is to fit the line with earlier prediction. With about $5 \%$ earlier fitting of the generation KNEE, the delay problem can be solved. Emulation with $2.0 \mathrm{~s}$ gait period has been conducted, and result showed a slight leading of the actual KNEE output, about $5 \%$ on average.

Another way is to use a faster motor to overcome the delay problem. However a faster motor is usually either heavy or with less torque. This could be dangerous to the user with mobility problem. That is why we introduced the foot switch method and consider it as the most appropriate implementation for HIP-KNEE control.

\section{CONCLUSION}

In the hardware aspect, the designed PKO provides a low-profile but high performance for gait assistance. With replaceable tailor-made knee orthosis, the fitting is adjustable to different wearers. The PKO system is only 2.4 $\mathrm{kg}$ load on one leg. It is able to add more actuators to enhance the system ability such as the ankle joint. The integration of the HIP actuator is feasible by the combination of the grounded HIP actuator.

Regarding the control, it is simple yet useful to swing the knee at right time to assist motion. The simple HIP-KNEE model reduces computational effort and enables designers understand parameters initiatively for adjusting them if necessary. Because no sensor for detecting GRF and other joint angles of the wearer is needed, this method has the minimal sensor number and attachment. This is also the merit of HIP-KNEE control.

From the results, it showed that HIP-KNEE control with footswitch is more natural and robust to generate proper KNEE according to the input HIP. The flexion of KNEE starts earlier than normal people to provide assistive torque. The synchronization is done by HIP to KNEE. It is possible to do the HAND to HIP and KNEE to ANKLE in the future.

In consequence, a new algorithm named HIP-KNEE control is proposed based on the HIP to estimate the desired KNEE. It is able to function for the PKO system and similar 1 DOF devices. It reduces the required sensor number and performs close function as normal human. Under simulation, emulation and testing on human subject, results show the control is workable to wearers during swing and stance phases in the gait. The control is hopefully applied to other joint for performing similar function.

\section{ACKNOWLEDGMENT}

This work is supported by the Start-up Support Scheme, Shenzhen Research Institute, The Chinese University of Hong Kong.

\section{REFERENCES}

[1] A. M. Dollar and H. Herr, "Lower extremity exoskeletons and active orthoses: challenges and state-of-the-art," IEEE Transactions on Robotics, vol. 24, no. 1, pp. 144-158, 2008.

[2] A. Tsukahara, Y. Hasegawa, and Y. Sankai, "Gait support for complete spinal cord injury patient by synchronized leg-swing with HAL," Proc. of the 2011 IEEE/RSJ Int'l Conf. on Intelligent Robots and Systems, San Francisco, CA, Sep. 2011, pp. 1737-1742.

[3] A. Goffer, "Gait-locomotor apparatus," U.S. Patent 7153 242, December 26, 2006.

[4] Ekso Bionics. http://www.eksobionics.com/ekso.

[5] R. J. Farris, H. A. Quintero, and M. Goldfarb, "Preliminary evaluation of a powered lower limb orthosis to aid walking in paraplegic individuals," IEEE Transactions on Neural Systems and Rehabilitation Engineering, vol. 19, no. 6, pp. 652-659, 2011.

[6] H. Ma, W. Y. Lai, W. H. Liao, T. P. Fong, and K. M. Chan, "Design and control of a powered knee orthosis for gait assistance," IEEE/ASME Int. Conf. on Advanced Intelligent Mechatronics, Wollongong, Australia, pp. 816-821, Jul. 2013.

[7] D. A. Winter, The Biomechanics and Motor Control of Human Gait: Normal, Elderly and Pathological, Second Edition, University of Waterloo Press, 1991.

[8] J. Rose and J. G. Gamble Human walking. Philadelphia: Lippincott Williams \& Wilkins, 2006, ch. 3.

[9] I. Diaz, J. J. Gil, and E. Sanchez, "Lower-limb robotic rehabilitation: literature review and challenges,” Journal of Robotics, vol. 2011, pp. 1-11, 2011.

[10] K. Suzuki, G. Mito, H. Kawamoto, Y. Hasegawa, and Y. Sankai, "Intention-based walking support for paraplegia patients with Robot Suit HAL,” Advanced Robotics, vol. 2, no. 12, pp. 1441-1469, 2007. 